\title{
Producción de arrabio en los países de la Unión Europea ${ }^{(*)}$
}

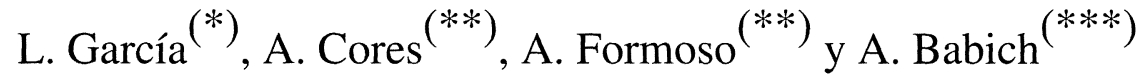

Resumen El aumento continuo de la competitividad en la industria siderúrgica hace necesaria la optimización de los recursos y materias primas empleados. Este hecho afecta de igual forma a la fabricación de arrabio en horno alto y a su principal combustible, el coque metalúrgico. De ahí la gran importancia que adquiere el desarrollo de técnicas que faciliten mejoras en los índices de operación del horno alto. En este trabajo se analizan los últimos desarrollos aplicados al proceso del horno alto en los países de la Unión Europea.

Palabras clave: Horno alto. Consumo de coque. Productividad. Inyección de combustible por toberas. Inyección de carbón.

\section{Pig iron production in the countries of European Community}

\begin{abstract}
The incessant increase of the competitivy of the metallurgical industry implies the necessity of the optimization of the resources and raw materials used. This affects in the same way the ironmaking in blast furnace and its principal fuel, metallurgical coke. It is from here the importance of the development of the techniques which facilitate improvements in the operation parameters in the blast furnace. This article is dedicated to the latest developments applicated on blast furnace process in the countries of European Community.
\end{abstract}

Keywords: Blast furnace. Coke consumption. Productivity. Fuels injection by tuyeres. Coal injection.

\section{INTRODUCCIÓN}

La etapa moderna de desarrollo de la industria siderúrgica está caracterizada, fundamentalmente, por la utilización de tecnologías de vanguardia, teniendo en cuenta la economía de recursos naturales y la protección del medio ambiente. La fabricación de arrabio en el horno alto no es ajena a esta tendencia y sus operadores, para competir con éxito en el futuro, deberán lograr los objetivos siguientes:

a) Incremento de la eficiencia del horno alto, que se logra con:

- elevada productividad específica,

(•) Trabajo recibido el día 28 de febrero de 1997

(*) Unión de Empresas de Recuperación de Materias Primas. $1^{a}$ Avda., 1604. Miramar. 11300 Ciudad de La Habana (Cuba).

(**) Centro Nacional de Investigaciones Metalúrgicas (CSIC). Avda. Gregorio del Amo, 8. 28040-Madrid (España).

(***) Universidad Estatal Tecnológica de Donetsk. Avda. Arteoma, 58. 340066 Donetsk (Ucrania).
- reducción de la relación coque/combustible,

- operación estable,

- elevado aprovechamiento.

b) Calidad constante del arrabio, como resultado de:

- la estabilidad en los procesos de reducción y fusión,

- la buena permeabilidad del crisol.

c) Prolongación del tiempo de campaña del horno, como resultado de:

- alargar la vida útil de los refractarios en el crisol y en el vientre,

- desprendimiento mínimo de materiales de carga y de pegotes de las paredes del horno,

- descenso regular de la carga.

d) Reducir el costo de la carga mediante:

- uso de materiales férreos de bajo coste y alta calidad,

- reducción de los costos de coque manteniendo su calidad y consistencia, 
- disminución del consumo de coque mediante la inyección por toberas de combustibles auxiliares, en particular empleando caudales elevados de inyección de carbón (pulverizado o granular).

En este trabajo se realiza un análisis de la producción de arrabio en la Unión Europea (UE) en los últimos años.

\section{PRODUCCIÓN DE ARRABIO EN HORNO ALTO}

La producción mundial de arrabio en 1996 fue de $521,2 \times 10^{6} \mathrm{t}$, de la que aproximadamente el 20 $\%$ se fabrica en los países de la UE. En la tabla I se ofrecen los datos de la producción de arrabio en la UE y en otras zonas geográficas ( 1 y 2 ). A escala mundial, se destaca el paulatino crecimiento de la producción en los países asiáticos, así como el brusco descenso en Europa Oriental como consecuencia de las transformaciones políticas y económicas ocurridas en la antigua URSS y en el denominado bloque socialista.
En Europa, como en el resto del mundo, en el próximo decenio el acero seguirá siendo el principal material que se empleará en la construcción de maquinaria y equipos, así como en la propia industria de la construcción y, por consiguiente, la demanda de productos siderúrgicos tenderá a crecer (3). La producción de acero en la UE durante 1995 fue de $155,8 \times 10^{6} \mathrm{t}$, de la que el $65,1 \%$ se obtiene mediante el procedimiento de horno alto-convertidor.

Por otra parte, la proporción de arrabio en la fabricación de acero en convertidor varía desde un $65 \%$ en Luxemburgo hasta el $86 \%$ en Francia y Finlandia. El promedio para la UE se mantiene en el $80 \%$, lo que está relacionado con la escasez de chatarra de acero y con las estrictas limitaciones de muchas calidades de acero según el contenido de elementos de aleación.

La industria siderúrgica es una de las mayores consumidoras de energía; en Japón, por ejemplo, esta industria consume el $13-15 \%$ de toda la energía del país (4).

En la tabla II se ofrece el balance energético promedio de la industria siderúrgica en 1994 para los siguientes países de la UE: Alemania, Bélgica, España, Francia, Holanda y Reino Unido. En las

TABla I.- Producción de arrabio, $10^{6} \mathrm{t}$

TABLE I.-Pig iron production, $10^{6} t$

\begin{tabular}{|l|r|r|r|r|r|r|r|r|}
\hline País, zona & \multicolumn{1}{|c|}{1989} & \multicolumn{1}{c|}{1990} & \multicolumn{1}{c|}{1991} & 1992 & 1993 & 1994 & 1995 & 1996 \\
\hline Alemania & 29,8 & 27,4 & 27,8 & 26,3 & 25,8 & 28,5 & 30,0 & 27,7 \\
Francia & 14,6 & 13,7 & 13,4 & 12,8 & 11,8 & 12,9 & 13,2 & 12,4 \\
Reino Unido & 12,6 & 12,2 & 11,9 & 11,9 & 11,5 & 12,0 & 12,2 & 12,8 \\
Italia & 11,4 & 10,5 & 10,5 & 8,7 & 9,5 & 9,5 & 11,7 & 10,3 \\
Bélgica & 7,4 & 8,3 & 8,3 & 8,2 & 8,1 & 9,0 & 9,3 & 8,7 \\
Holanda & 5,2 & 4,7 & 4,7 & 4,9 & 5,5 & 5,5 & 5,5 & 5,5 \\
España & 5,6 & 5,6 & 5,6 & 5,1 & 5,3 & 5,5 & 5,1 & 4,1 \\
Austria & 3,7 & 3,4 & 3,5 & 3,1 & 3,1 & 3,4 & 3,9 & 3,4 \\
Suecia & 2,7 & 2,7 & 2,8 & 2,8 & 2,9 & 3,1 & 3,0 & 3,1 \\
Finlandia & 2,3 & 2,3 & 2,3 & 2,5 & 2,5 & 2,6 & 2,2 & 2,5 \\
Luxemburgo & 2,7 & 2,6 & 2,5 & 2,3 & 2,4 & 1,9 & 0,9 & 0,8 \\
Portugal & 0,4 & 0,3 & 0,3 & 0,4 & 0,4 & 0,4 & 0,4 & 0,4 \\
\hline Total UE & $\mathbf{9 8 , 6}$ & $\mathbf{9 3 , 7}$ & $\mathbf{9 3 , 6}$ & $\mathbf{8 9 , 0}$ & $\mathbf{8 8 , 8}$ & $\mathbf{9 4 , 3}$ & $\mathbf{9 7 , 5}$ & $\mathbf{9 1 , 8}$ \\
\hline Turquía & 5,5 & 5,4 & 5,2 & 5,1 & 4,9 & 4,6 & 4,4 & 5,3 \\
Otros E. Occ. & 2,5 & 1,4 & 0,3 & 0,3 & 0,2 & 0,2 & 0,3 & 0,7 \\
Europa Occ. & 106,6 & 100,5 & 99,1 & 94,4 & 94,9 & 99,1 & 102,2 & 97,8 \\
Europa Oriental & 146,4 & 141,3 & 114,2 & 106,2 & 90,9 & 80,5 & 87,5 & 85,1 \\
Norte América & 65,9 & 65,0 & 59,4 & 63,4 & 56,8 & 57,5 & 59,4 & 58,0 \\
Sur América & 27,8 & 28,6 & 30,0 & 30,7 & 29,8 & 31,5 & 32,2 & 31,8 \\
Asia & 179,1 & 188,7 & 200,0 & 204,0 & 211,7 & 223,2 & 234,7 & 236,1 \\
África & 11,1 & 11,6 & 12,6 & 11,7 & 8,7 & 9,4 & 8,6 & 8,4 \\
Oceanía & 6,3 & 6,7 & 6,2 & 7,0 & 7,4 & 7,5 & 8,1 & 8,0 \\
\hline Mundial & $\mathbf{5 4 3 , 0}$ & $\mathbf{5 4 2 , 4}$ & $\mathbf{5 2 1 , 5}$ & $\mathbf{5 1 7 , 4}$ & $\mathbf{5 0 0 , 2}$ & $\mathbf{5 0 8 , 7}$ & $\mathbf{5 2 9 , 5}$ & $\mathbf{5 2 1 , 2}$ \\
\hline
\end{tabular}


TABLA II.- Balance energético en la industria siderúrgica (por $t$ de acero líquido)

TABLE II.-Energy balance in the siderurgical industry (per t of crude steel)

\begin{tabular}{|c|c|c|c|c|c|c|c|c|c|}
\hline Sector de consumo & & $\begin{array}{l}\text { Prepara- } \\
\text { ción del } \\
\text { mineral }\end{array}$ & $\begin{array}{l}\text { Fabrica- } \\
\text { ción de } \\
\text { coque }\end{array}$ & $\begin{array}{l}\text { Fabrica- } \\
\text { ción de } \\
\text { arrabio }\end{array}$ & $\begin{array}{c}\text { Elaborac. } \\
\text { de acero } \\
\text { líquido }\end{array}$ & $\begin{array}{c}\text { Laminado } \\
\text { y } \\
\text { acabado }\end{array}$ & Otros & TOTAL & $\%$ \\
\hline Carbón crudo & GJ & 0,2024 & 10,7092 & 2,1258 & 0,0156 & \multirow{3}{*}{0,0001} & 0,0191 & 13,0721 & 35,96 \\
\hline Coque & “ & 1,0274 & 0,0218 & 8,6121 & 0,0236 & & 0,0058 & 9,6908 & 26,67 \\
\hline Otros combust. sólidos & “ & 0,0497 & 0,3946 & 0,0060 & 0,0061 & & 0,0457 & 0,5021 & 1,38 \\
\hline Gas de batería & “ & 0,0955 & 0,4928 & 0,3300 & 0,0444 & 0,4460 & 0,3156 & 1,7243 & 4,74 \\
\hline Gas de horno alto & “ & 0,0327 & 0,2392 & 1,0871 & 0,0007 & 0,0924 & 1,0292 & 2,4813 & 6,83 \\
\hline Gas de LD & “ & 0,0008 & 0,0232 & 0,0301 & 0,0008 & 0,0509 & 0,0617 & 0,1675 & 0,46 \\
\hline Gas natural & “ & 0,0374 & \multirow[t]{2}{*}{0,0010} & 0,0865 & 0,1678 & 1,1461 & 0,3088 & 1,7476 & 4,81 \\
\hline Otros combus. gaseosos & “ & \multirow{5}{*}{0,3181} & & 0,0009 & 0,0093 & 0,0314 & 0,0069 & 0,0485 & 0,13 \\
\hline Fuel oil & “ & & 0,0023 & 0,5533 & 0,0029 & 0,1160 & 0,1241 & 0,7986 & 2,20 \\
\hline Otros combus. líquidos & “ & & 0,0057 & 0,0006 & 0,0023 & 0,0012 & 0,0093 & 0,0191 & 0,05 \\
\hline Electricidad & “ & & \multirow[t]{2}{*}{0,1122} & 0,3237 & 1,5647 & 1,5263 & 0,4354 & 4,2804 & 11,78 \\
\hline Oxígeno & “ & & & 0,1256 & 0,3063 & 0,0044 & 0,0009 & 0,4372 & 1,20 \\
\hline Vapor & “ & 0,0021 & 0,0959 & 0,3133 & 0,0486 & 0,1404 & 0,7781 & 1,3784 & 3,79 \\
\hline Producción bruta & GJ & 1,7661 & 12,0979 & 13,5950 & 2,1931 & 3,5552 & 3,1346 & 36,3419 & 100,00 \\
\hline Coque producido & GJ & & 7,8895 & \multirow{4}{*}{3,0738} & \multirow{7}{*}{0,2849} & & \multirow{7}{*}{$\begin{array}{l}0,8752 \\
0,0263\end{array}$} & 7,8895 & 50,16 \\
\hline Gas CO recuperado & “ & & 1,7048 & & & & & 1,7048 & 10,84 \\
\hline Gas horno alto recup. & “ & & \multirow{5}{*}{0,3967} & & & & & 3,0738 & 19,55 \\
\hline Gas de LD recobrado & “ & & & & & & & 0,2849 & 1,81 \\
\hline Recuper. de alquit. y otros & “" & & & 0,0057 & & & & 0,4024 & 2,56 \\
\hline Electricidad producida & “ & & & 0,0560 & & & & 0,9312 & 5,92 \\
\hline Oxígeno producido & “ & & & & & & & 0,0263 & 0,17 \\
\hline Vapor & 6 & 0,0346 & 0,0156 & 0,0029 & 0,0349 & 0,0010 & 1,3242 & 1,4132 & 8,99 \\
\hline Total recobrado & GJ & 0,0346 & 10,0066 & 3,1384 & 0,3198 & 0,0010 & 2,2257 & 15,7261 & 100,00 \\
\hline \multirow[t]{2}{*}{ Energía neta empleada } & GJ & 1,7315 & 2,0913 & 10,4566 & 1,8733 & 3,5542 & 0,9089 & 20,6158 & \\
\hline & $\%$ & 8,40 & 10,14 & 50,72 & 9,09 & 17,24 & 4,41 & 100,00 & \\
\hline
\end{tabular}

etapas principales del proceso siderúrgico se destaca que la fabricación de arrabio es la mayor consumidora de combustible (más del $50 \%$ ); por otra parte, en el horno alto el coque es el combustible más utilizado, lo que ejercerá una influencia determinante en los costos de fabricación de las posteriores etapas de procesamiento. Se estima que entre un 30-35\% del coste del arrabio corresponde al consumo de combustible; por tanto, lograr disminuir el consumo de coque ha sido, desde siempre, una de las principales tareas de los operadores e investigadores (5).

El análisis de los índices técnico-económicos de los hornos altos de la UE confirma la elevada eficiencia de operación (6). En la tabla III se incluyen datos promediados por país del funcionamiento de los hornos de la UE. El consumo de coque, en la mayoría de los países, es de 350-400 kg/ta, con algunas excepciones de países con tasas que superan los $400 \mathrm{~kg} / \mathrm{ta}$. Se destaca que en Italia, desde 1989 a 1995, disminuyó el consumo de coque en $86 \mathrm{~kg} / \mathrm{ta}$, y en Francia y en Alemania en $50 \mathrm{~kg} / \mathrm{ta}$. Durante 1995 los menores consumos de coque se consiguieron en los hornos siguientes:

HA-A de HKM, en Huckingen

(Alemania)
HA-1 de SOLLAC, en Fos

(Francia) $325 \mathrm{~kg} / \mathrm{ta}$

HA-2 de SOLLAC, en Fos

(Francia) $325 \mathrm{~kg} / \mathrm{ta}$

HA-A de SIDMAR, en Gante

(Bélgica) $328 \mathrm{~kg} / \mathrm{ta}$

HA-B de HKM, en Huckingen

(Alemania) $330 \mathrm{~kg} / \mathrm{ta}$

La productividad de los hornos ha experimentado una mejora en los últimos seis años. En Holanda aumenta desde 2,15 hasta 2,67 t/m $\mathrm{m}^{3} \cdot 24 \mathrm{~h}(24 \%)$, también Italia y Suecia alcanzan productividades superiores a $2,6 \mathrm{t} / \mathrm{m}^{3} \cdot 24 \mathrm{~h}$ con incrementos en este período del 16 y $13 \%$, respectivamente. Los hornos con mejores productividades durante 1995 han sido:

HA-2 de Raahe Steel, en Rautaruukki

(Finlandia) $3,05 \mathrm{t} / \mathrm{m}^{3} \cdot 24 \mathrm{~h}$ HA-2 de SSAB, en Luleâ (Suecia) $\quad 3,05 \mathrm{t} / \mathrm{m}^{3} \cdot 24 \mathrm{~h}$ HA-A de HKM, en Huckingen

(Alemania) $2,92 \mathrm{t} / \mathrm{m}^{3} \cdot 24 \mathrm{~h}$

HA-6 de Hoogovens, en IJmuiden (Holanda) $2,92 \mathrm{t} / \mathrm{m}^{3} \cdot 24 \mathrm{~h}$ 
TABLA III.- Índices medios de operación de los hornos altos en 1995

TABLE III.-Mean parameters operation of blast furnaces in 1995

\begin{tabular}{|l|c|r|r|r|r|r|r|r|r|r|r|r|r|}
\hline & Unidad & \multicolumn{1}{c|}{$\mathrm{A}$} & \multicolumn{1}{c|}{$\mathrm{F}$} & \multicolumn{1}{c|}{$\mathrm{RU}$} & \multicolumn{1}{c|}{$\mathrm{I}$} & $\mathrm{B}$ & $\mathrm{H}$ & $\mathrm{E}$ & $\mathrm{Au}$ & $\mathrm{S}$ & $\mathrm{Fi}$ & $\mathrm{L}$ & $\mathrm{P}$ \\
\hline Hornos en operación & $\mathrm{U}$ & 20 & 11 & 8 & 5 & 8 & 2 & 6 & 5 & 4 & 3 & 1 & 1 \\
Diámetro crisol & $\mathrm{m}$ & 10,2 & 9,2 & 10,0 & 10,8 & 9,1 & 12,4 & 8,8 & 8,4 & 7,6 & 7,35 & 9,2 & 6,5 \\
Volumen útil & $\mathrm{m}^{3}$ & 1988 & 1716 & 1941 & 2196 & 1570 & 3059 & 1483 & 1392 & 971 & 821 & 1790 & 670 \\
Productividad & $\mathrm{t} / \mathrm{m}^{3} \cdot 24 \mathrm{~h}$ & 2,13 & 2,18 & 2,36 & 2,63 & 2,25 & 2,67 & 1,90 & 1,78 & 2,60 & 2,85 & 1,64 & 1,88 \\
Carga férrea: & & & & & & & & & & & & & \\
-miner. y otras dicion. & $\%$ & 8,0 & 12,1 & 23,0 & 14,3 & 5,6 & 2,7 & 5,4 & 13,4 & 0 & 0 & 1,7 & 0 \\
-sínter & $\%$ & 65,0 & 87,6 & 69,6 & 78,9 & 63,6 & 47,9 & 68,4 & 58,5 & 15,7 & 87,2 & 98,3 & 68,3 \\
-pelets & $\%$ & 27,0 & 0,3 & 7,4 & 6,8 & 30,8 & 49,4 & 26,2 & 28,1 & 84,3 & 12,8 & 0 & 31,7 \\
Consumo de coque & $\mathrm{kg} / \mathrm{ta}$ & 359 & 351 & 394 & 353 & 382 & 357 & 473 & 426 & 383 & 375 & 462 & 500 \\
Caudal de fuel oil & $\mathrm{kg} / \mathrm{ta}$ & 51 & 4 & 54 & 15 & 2 & 0 & 26 & 49 & 0 & 74 & 59 & 0 \\
Caudal CP & $\mathrm{kg} / \mathrm{ta}$ & 63 & 126 & 43 & 130 & 123 & 141 & 0 & 0 & 80 & 0 & 0 & 0 \\
Temperatura viento & ${ }^{\circ} \mathrm{C}$ & 1168 & 1184 & 1084 & 1177 & 1109 & 1195 & 1088 & 1097 & 1079 & 1070 & 1128 & 918 \\
Oxígeno en el viento & $\%$ & 22,7 & 22,1 & 24,3 & 24,1 & 24,1 & 23,5 & 21,2 & 21,1 & 22,9 & 24,2 & 23,0 & 21,0 \\
Temperatura llama & ${ }^{\circ} \mathrm{C}$ & 2161 & 2147 & 2110 & 2128 & 2223 & 2164 & 2191 & 2136 & 2185 & 2242 & $\mathrm{ND}$ & 2182 \\
Conten. en el arrabio: & & & & & & & & & & & & \\
-silicio & $\%$ & 0,51 & 0,52 & 0,55 & 0,62 & 0,58 & 0,42 & 0,64 & 0,75 & 0,49 & 0,47 & 0,50 & 0,52 \\
-azufre & $\%$ & 0,052 & 0,036 & 0,025 & 0,022 & 0,028 & 0,036 & 0,036 & 0,049 & 0,057 & 0,061 & 0,021 & 0,068 \\
Temperatura del arrabio & ${ }^{\circ} \mathrm{C}$ & 1467 & 1476 & 1496 & 1495 & 1475 & 1511 & 1490 & 1444 & 1470 & 1467 & 1491 & 1444 \\
Volumen de escoria & $\mathrm{kg} / \mathrm{ta}$ & 267 & 297 & 288 & 303 & 271 & 223 & 301 & 304 & 153 & 203 & 509 & 258 \\
\hline
\end{tabular}

A = Alemania; F = Francia; RU = Reino Unido; I = Italia; B = Bélgica; H = Holanda; $\mathrm{E}=$ España; Au = Austria; $\mathrm{S}=\mathrm{Suecia}$;

$\mathrm{Fi}=$ Finlandia $; \mathrm{L}=$ Luxemburgo $; \mathrm{P}=$ Portugal

HA-A de SIDMAR, en Gante

(Bélgica)

$2,84 \mathrm{t} / \mathrm{m}^{3} \cdot 24 \mathrm{~h}$

En las tablas IV y V se muestran las características de algunos hornos que se mencionan en este trabajo. En los hornos se realizan inyecciones de car- bón pulverizado o de fuel oil con el objetivo principal de reducir el consumo de coque por tonelada de arrabio producido.

Estos éxitos notables se han alcanzado debido a una serie de medidas, entre las que destacan: uso de materias primas férreas y coque metalúrgico de alta

TABLA IV.- Índices de operación de hornos altos con inyección de carbón en 1995

TABLE IV.- Parameters operation of blast furnaces with coal injection in 1995

\begin{tabular}{|c|c|c|c|c|c|c|c|}
\hline Índices & $\begin{array}{c}\text { Thyssen } \\
\text { Schwelgern } \\
\text { HA-1 } \\
\text { Alemania }\end{array}$ & $\begin{array}{c}\text { Krupp Hoesch } \\
\text { Westfalenhütte } \\
\text { HA-7 } \\
\text { Alemania }\end{array}$ & $\begin{array}{c}\text { Sollac } \\
\text { Dunkerque } \\
\text { HA-4 } \\
\text { Francia }\end{array}$ & $\begin{array}{l}\text { Hoogovens } \\
\text { IJmuiden } \\
\text { HA-6 } \\
\text { Holanda }\end{array}$ & $\begin{array}{c}\text { Ilva } \\
\text { Taranto } \\
\text { HA-2 } \\
\text { Italia }\end{array}$ & $\begin{array}{c}\text { SIDMAR } \\
\text { Gent } \\
\text { HA-A } \\
\text { Bélgica }\end{array}$ & $\begin{array}{l}\text { British Steel } \\
\text { Scunthorpe Q. } \\
\text { Victoria, R.U. }\end{array}$ \\
\hline Diám. de crisol, m & 13,6 & 10,2 & 14,0 & 11,0 & 10,34 & 10,0 & 9,0 \\
\hline Volumen útil, $\mathrm{m}^{3}$ & 3.796 & 1.875 & 3.648 & 2.328 & 1.971 & 1.776 & 1.537 \\
\hline Product., $\mathrm{t} / \mathrm{m}^{3} \cdot 24 \mathrm{~h}$ & 2,18 & 2,13 & 2,40 & 2,92 & 2,70 & 2,84 & 2,37 \\
\hline $\begin{array}{l}\text { Carga férrea, } \%: \\
\text { - miner. de hierro y otras }\end{array}$ & & & & & & & \\
\hline adiciones & 9,8 & 14,6 & 23,8 & 2,30 & 16,5 & 0,5 & 16,3 \\
\hline - sínter & 71,0 & 59,3 & 76,2 & 48,4 & 76,9 & 92,5 & 72,7 \\
\hline - pelets & 19,2 & 26,1 & 0 & 49,3 & 6,6 & 7,0 & 11,0 \\
\hline Cons. de coque, $\mathrm{kg} / \mathrm{ta}$ & 370 & 361 & 333 & 339 & 332 & 328 & 352 \\
\hline Carbón inyect., kg/ta & 133 & 150 & 152 & 161 & 170 & 164 & 146 \\
\hline Volátil. en carbón, \% & 24,4 & 29,9 & 14,4 & 38,8 & 27,0 & 29,9 & 18,0 \\
\hline Oxígeno en viento, $\%$ & 23,03 & 24,44 & 23,16 & 25,9 & 25,2 & 24,0 & 26,0 \\
\hline Temp. del viento, ${ }^{\circ} \mathrm{C}$ & 1.187 & 1.159 & 1.192 & 1.140 & 1.196 & 1.191 & 1.121 \\
\hline Temp. de llama, ${ }^{\circ} \mathrm{C}$ & 2.169 & $\mathrm{ND}$ & 2.129 & 2.109 & 2.133 & 2.239 & 2.121 \\
\hline Cont. en arrabio, \%: & & & & & & & \\
\hline - silicio & 0,42 & 0,66 & 0,34 & 0,41 & 0,70 & 0,37 & 0,62 \\
\hline - azufre & 0,033 & 0,067 & 0,029 & 0,035 & 0,021 & 0,021 & 0,024 \\
\hline Temp. del arrabio, ${ }^{\circ} \mathrm{C}$ & 1.492 & 1.489 & 1.486 & 1.505 & 1.499 & 1.493 & 1.481 \\
\hline Vol. de escoria, $\mathrm{kg} / \mathrm{ta}$ & 289 & 266 & 293 & 224 & 299 & 279 & 278 \\
\hline
\end{tabular}


TABLA V.- Índices de operación de hornos altos con inyección de fuel oil en 1995

TABLE V.-Parameters operation of blast furnaces with oil injection in 1995

\begin{tabular}{|c|c|c|c|c|c|c|c|}
\hline Índices & $\begin{array}{c}\text { HKM } \\
\text { Huckingen, } \\
\text { HA-A, } \\
\text { Alemania }\end{array}$ & $\begin{array}{c}\text { HKM } \\
\text { Huckingen, } \\
\text { HA-B, } \\
\text { Alemania }\end{array}$ & $\begin{array}{l}\text { Sthalwerke } \\
\text { Bremen, } \\
\text { HA-2, } \\
\text { Alemania }\end{array}$ & $\begin{array}{l}\text { Sthalwerke } \\
\text { Bremen, } \\
\text { HA-3, } \\
\text { Alemania }\end{array}$ & $\begin{array}{c}\text { VA } \\
\text { Linz, } \\
\text { HA-A, } \\
\text { Austria }\end{array}$ & $\begin{array}{c}\text { Raahe Steel } \\
\text { Rautarukki, } \\
\text { HA-2, } \\
\text { Finlandia }\end{array}$ & $\begin{array}{c}\text { British Steel } \\
\text { Llanwern, } \\
\text { HA-3, } \\
\text { Reino Unido }\end{array}$ \\
\hline Diám. de crisol, m & 10,3 & 10,3 & 12,0 & 9,2 & 10,5 & 7,2 & 11,20 \\
\hline Volumen útil, $\mathrm{m}^{3}$ & 2.081 & 2.226 & 2.776 & 1.424 & 2.454 & 836 & 2.289 \\
\hline Product., $\mathrm{t} / \mathrm{m}^{3} \cdot 24 \mathrm{~h}$ & 2,92 & 2,30 & 2,15 & 2,22 & 2,22 & 3,44 & 2,53 \\
\hline $\begin{array}{l}\text { Carga férrea, \%: } \\
\text { - miner. de hierro y otras }\end{array}$ & & & & & & & \\
\hline adiciones & 6,6 & 8,1 & 4,3 & 7,7 & 21,2 & 0 & 19,3 \\
\hline - sínter & 67,2 & 67,0 & 47,6 & 6,3 & 45,0 & 65,9 & 79,0 \\
\hline - pelets & 26,2 & 24,9 & 49,1 & 86,0 & 33,8 & 34,1 & 1,7 \\
\hline Cons. de coque, $\mathrm{kg} / \mathrm{ta}$ & 321 & 330 & 334 & 337 & 411 & 372 & 390 \\
\hline Caud. fuel oil, $\mathrm{kg} / \mathrm{ta}$ & 117 & 108 & 123 & 122 & 56 & 76 & 91 \\
\hline Oxígeno en viento, $\%$ & 25,15 & 24,42 & 22,45 & 21,12 & 21,20 & 24,50 & 25,45 \\
\hline Temp. del viento, ${ }^{\circ} \mathrm{C}$ & 1.249 & 1.221 & 1.193 & 1.130 & 1.181 & 1.097 & 1.025 \\
\hline Temp. de llama, ${ }^{\circ} \mathrm{C}$ & ND & ND & 2.011 & 1.956 & 2.140 & 2.260 & 2.066 \\
\hline Cont. en arrabio, $\%$ : & & & & & & & \\
\hline - silicio & 0,50 & 0,66 & 0,40 & 0,52 & 0,50 & 0,46 & 0,62 \\
\hline - azufre & 0,047 & 0,044 & 0,065 & 0,069 & 0,040 & 0,052 & 0,018 \\
\hline Temp. del arrabio, ${ }^{\circ} \mathrm{C}$ & 1.498 & 1.482 & 1.452 & 1.451 & 1.466 & 1.471 & 1.524 \\
\hline Vol. de escoria, $\mathrm{kg} / \mathrm{ta}$ & 254 & 248 & 242 & 179 & 304 & 203 & 318 \\
\hline
\end{tabular}

calidad, introducción eficaz y rápida de la inyección de carbón pulverizado (ICP), aumento de la temperatura del viento, optimización de los regímenes de escoria y gasodinámico del proceso, control automático, etc. Algunas de estas medidas serán analizadas en este trabajo, otras han sido tratadas en (7 y 8$)$.

\section{CARGA DEL HORNO ALTO}

\subsection{Materiales férreos}

En la figura 1 se muestra la evolución que han tenido los componentes férreos de la carga en los hornos altos de la UE durante el período 19801995. La carga está compuesta básicamente por los aglomerados, sínter y pelets. La proporción de mineral de hierro clasificado (en trozos) y otras adiciones de hierro en la carga de los hornos es, como regla, del 5-15\% y en el Reino Unido del 20-24\%. Solamente en el HA-1 de British Steel, en Redcar, es superior al $30 \%(545 \mathrm{~kg} / \mathrm{ta})$. En países como Holanda, Finlandia, Luxemburgo y Suecia, así como en algunos hornos de otros países, en la carga no se utiliza prácticamente mineral de hierro.

El sínter es el principal componente de la carga del horno. En la tabla VI se incluyen datos de la composición química y de la producción de sínter en la UE en 1995. Debido a condicionamientos económicos y ecológicos, se ha procedido al cierre de algunas fábricas de sinterización, lo que supone un

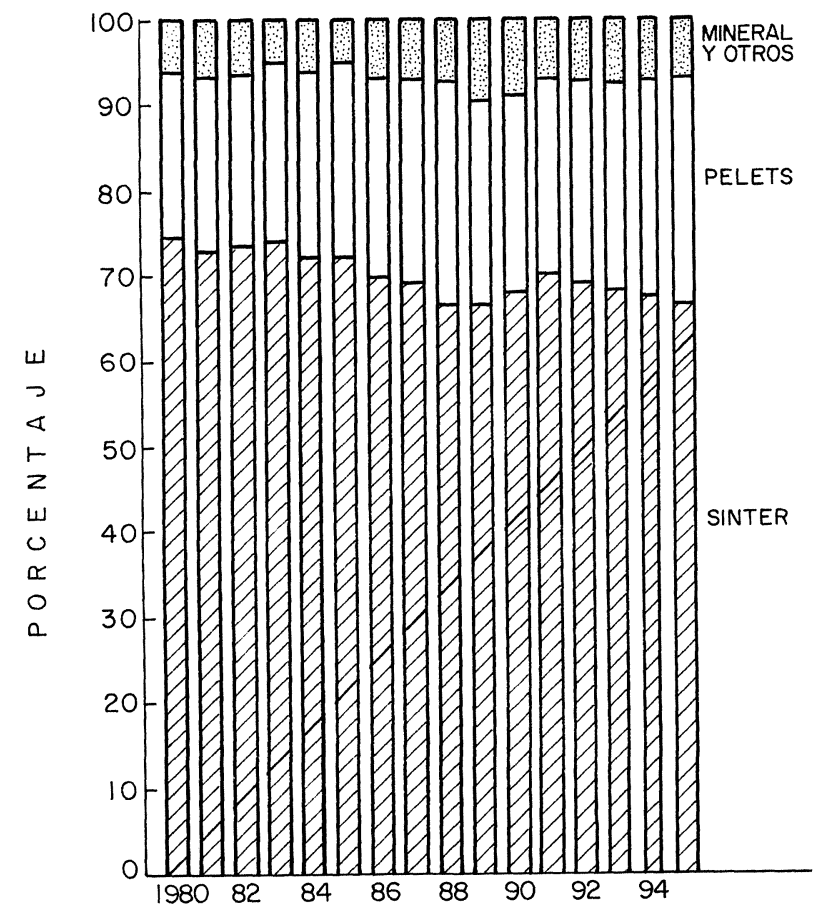

FIG. 1.- Proporción de los componentes férreos en la carga de los hornos altos de la UE.

FIG. 1.- Proportion of the iron components in the burden of the EU blast furnaces.

incremento de la proporción de pelets en la carga del horno.

Las mayores proporciones de pelets en la carga se utilizan en Suecia (81\%) y Holanda (49\%), 
TABLA VI.- Datos de operación de fábricas de sínter en 1995

TABLE VI.- Operating date of sinter plant in 1995

\begin{tabular}{|l|c|r|c|c|c|c|c|c|c|}
\hline \multirow{2}{*}{ País } & \multicolumn{7}{|c|}{ Composición química, \% } & \multirow{2}{*}{$\begin{array}{c}\text { Producción } \\
\times 106 \mathrm{t}\end{array}$} \\
\cline { 2 - 9 } & $\mathrm{Fe}_{\mathrm{t}}$ & $\mathrm{Fe}^{++}$ & $\begin{array}{c}\mathrm{Grado} \\
\text { oxid. }\end{array}$ & $\mathrm{SiO}_{2}$ & $\mathrm{CaO}$ & $\mathrm{IB}$ & $\mathrm{MgO}$ & $\mathrm{Al}_{2} \mathrm{O}_{3}$ & \\
\hline Austria & 49,3 & 4,5 & 97,0 & 7,3 & 15,4 & 2,1 & 3,3 & 1,4 & 2,16 \\
Bélgica & 59,1 & 3,9 & 97,8 & 5,2 & 7,4 & 1,4 & 1,8 & 1,3 & 5,70 \\
Finlandia & 62,0 & 9,0 & 94,9 & 3,7 & 5,8 & 1,6 & 1,8 & 0,6 & 2,23 \\
Francia & 57,7 & 4,7 & 97,3 & 5,3 & 9,4 & 1,8 & 1,6 & 1,2 & 6,65 \\
Alemania & 57,8 & 5,2 & 97,0 & 5,3 & 9,6 & 1,8 & 1,8 & 1,1 & 10,9 \\
Italia & 56,4 & 4,7 & 97,2 & 5,8 & 9,9 & 1,7 & 1,7 & 1,3 & 11,9 \\
Luxemburgo & 51,0 & 6,0 & 95,9 & 8,5 & 11,9 & 1,4 & 1,3 & 2,9 & 1,99 \\
Holanda & 57,6 & 13,0 & 92,5 & 4,5 & 10,5 & 2,4 & 1,5 & 1,4 & 4,25 \\
Portugal & 58,1 & 6,5 & 96,3 & 5,2 & 8,4 & 1,6 & 1,4 & 1,4 & 0,55 \\
España & 57,1 & 4,5 & 97,4 & 5,4 & 9,6 & 1,8 & 1,6 & 1,4 & 4,75 \\
Suecia & 62,2 & 14,0 & 92,4 & 3,5 & 6,0 & 1,7 & 1,4 & 0,5 & 0,60 \\
R. Unido & 56,7 & 3,7 & 97,8 & 5,8 & 9,2 & 1,6 & 1,8 & 1,4 & 5,34 \\
\hline
\end{tabular}

$\mathrm{IB}=\mathrm{CaO} / \mathrm{SiO}_{2}$

aunque en Alemania, Bélgica, España, Portugal y Austria se emplean proporciones comprendidas entre el 26 y el $32 \%$; en Francia, Italia y Reino Unido las proporciones son relativamente bajas (aprox. $7 \%$ ), y en Luxemburgo no se emplean pelets. Se destaca que Suecia incrementa considerablemente la proporción de pelets en la carga (desde un $47 \%$ en 1980 hasta el $81 \%$ en 1995), sin embargo, en el Reino Unido ocurre lo contrario, pues si en 1980-1990 se empleaba una media del 23 $\%$, a partir de 1991 disminuyó por debajo del $8 \%$. España mantuvo en este período bastante estabilidad en lo que respecta a los componentes de la carga del horno, donde son predominantes el sínter y los pelets (aprox. 70 y $24 \%$, respectivamente). En la tabla VII se muestra la composición química de pelets utilizados en hornos de España y Suecia.

El consumo de fundente, con raras excepciones, no es superior a 25-30 kg/ta. En Francia, Finlandia, Austria, y en algunos hornos altos de Bélgica,
España y Reino Unido, no se realizan este tipo de adiciones a la carga del horno.

La chatarra también encuentra poca utilización como componente de la carga. La empresa EKO (Alemania) utiliza en sus tres hornos entre 50 y 90 $\mathrm{kg} / \mathrm{ta}$, la empresa VA (Austria) en el HA-6 de su fábrica en Linz y en los HA-1 y 4 en Donawitz emplea entre 21 y $52 \mathrm{~kg} / \mathrm{ta}$, aunque el mayor consumo de chatarra durante 1995 se produjo en el HA-7 de Lorfonte, en Rombas, con 102 kg/ta.

\subsection{Coque}

La calidad del coque es muy importante debido a que con un bajo consumo, como consecuencia de la utilización de otros combustibles auxiliares, aumenta el tiempo de retención del coque en el horno ocasionando un aumento de la degradación del coque en el crisol, un empeoramiento de la eliminación de los álcalis y otras influencias negativas.

TABLA VII.- Composición química de los pelets

TABLE VII.- Chemical composition of pellets

\begin{tabular}{|l|ccccccccc|}
\hline \multirow{2}{*}{\multicolumn{1}{c|}{ Tipo }} & \multicolumn{10}{|c|}{ Composición química, \% } \\
\cline { 2 - 10 } & $\mathrm{Fe}$ & $\mathrm{SiO}_{2}$ & $\mathrm{Al}_{2} \mathrm{O}_{3}$ & $\mathrm{CaO}$ & $\mathrm{MgO}$ & $\mathrm{S}$ & $\mathrm{P}$ & $\mathrm{Na}_{2} \mathrm{O}$ & $\mathrm{K}_{2} \mathrm{O}$ \\
\hline LKAB (básicos) & 67,0 & 2,00 & - & - & 1,50 & - & 0,010 & 0,06 & 0,030 \\
LKAB (con fundente) & 66,3 & 1,94 & 0,81 & 1,61 & 0,37 & - & 0,06 & 0,02 & - \\
HISPANOBRAS (con & & & & & & & & & \\
fundente) & 65,9 & 2,37 & 0,44 & 2,53 & 0,06 & 0,05 & 0,026 & 0,01 & 0,012 \\
HISPANOBRAS (ácidos) & 65,2 & 5,10 & 0,42 & 0,75 & 0,20 & 0,08 & 0,012 & 0,03 & 0,018 \\
\hline
\end{tabular}


La composición química del coque debe cumplir los siguientes limites: cenizas $<10 \%$, azufre $<0,7$ $\%$, fósforo $<0,025 \%$ y álcalis $<0,28 \%$.

Con relación a las propiedades físico-mecánicas, el coque de tamaño superior a $25 \mathrm{~mm}$ debe cumplir los límites siguientes de resistencia a la abrasión:

- Ensayo MICUM, tamiz de $40 \mathrm{~mm}: \mathrm{M} 40 /+60=$ $87 \%$,

- Ensayo MICUM, tamiz de $10 \mathrm{~mm}$ : M10/+60 = $5,5 \%$,

- Ensayo IRSID, tamiz de $40 \mathrm{~mm}: \mathrm{I} 40 /+20=55 \%$,

- Ensayo IRSID, tamiz de $10 \mathrm{~mm}: \mathrm{I10} /+20=16 \%$.

Para las propiedades de reactividad:

- Índice de reactividad del coque: $\mathrm{CRI}=24 \%$,

- Resistencia del coque después de reaccionar: $\mathrm{CSR}=70 \%$.

\section{OPERACIÓN DEL HORNO ALTO}

\subsection{Distribución de los materiales de carga y de la corriente gaseosa}

Cuanto mayor sea la tasa de combustible auxiliar que se inyecta al horno alto, menor es el consumo de coque y, por tanto, mayor es la relación mineral/coque que se carga al horno.

La disminución del consumo de coque, si no se acompaña con las medidas adecuadas para mantener las condiciones gasodinámicas de la fusión, conlleva inevitablemente a perturbaciones en la marcha del horno y empeora sus índices de operación (9).

La distribución adecuada de los materiales en el horno se consigue al garantizar una permeabilidad elevada a los gases en el centro del horno con un moderado desarrollo de la corriente gaseosa por la periferia, que excluya grandes pérdidas de calor y la formación de pegotes, así como la creación de condiciones para el desarrollo de la reducción indirecta.

Para alcanzar este objetivo, en Francia se cargan los materiales gruesos en el centro del horno, y los finos hacia la periferia. Esto permite desarrollar una corriente gaseosa central con cantidad suficiente de mineral en el centro, garantizando, de tal forma, una buena utilización de la energía reductora de los gases y un consumo bajo de combustible.

La técnica de la carga central del coque se utiliza cuando se carga el horno con una relación elevada mineral/coque, y es importante para conseguir una zona cohesiva con la forma óptima de $\mathrm{V}$ invertida, que permite lograr una mejora de la permeabilidad y distribución del flujo de gas en el horno. Esta técnica ha sido desarrollada por Kobe Steel (10) y es uno de los medios más efectivos para controlar la distribución de la carga (Fig. 2). El coque

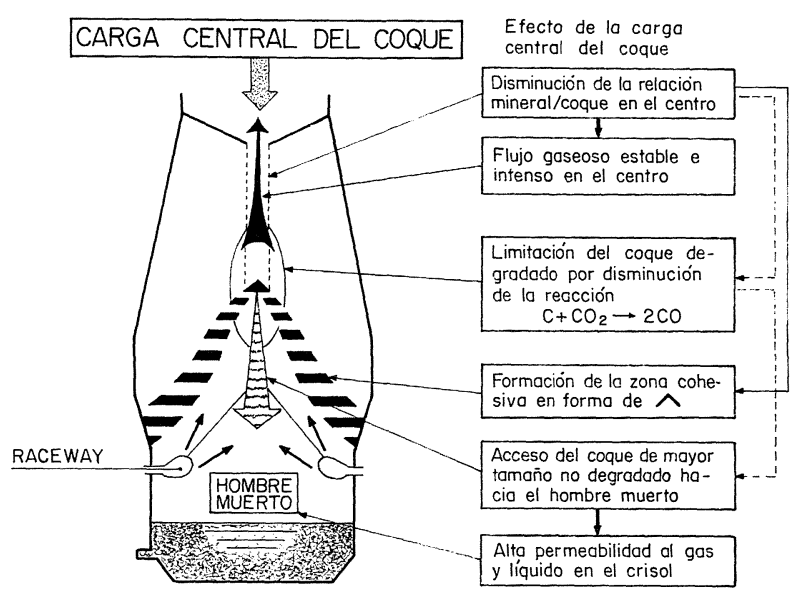

FIG. 2.- Nuevo concepto del horno alto con carga central de coque.

FIG. 2.- New concept of blast furnace with central coke charging.

que se carga en un área central estrecha forma la columna central de coque en el horno, la cual origina una zona cohesiva con forma de $\mathrm{V}$ invertida. La columna no está sometida a la reacción con mineral, puesto que no hay mineral en el centro del horno. Esta columna también forma el "hombre muerto", mejorando la permeabilidad del gas y líquido en la parte más baja del horno.

La carga central de coque se emplea en los hornos de Japón, Holanda, Alemania y otros países.

A continuación, como ejemplo, se va a analizar el funcionamiento de dos hornos altos: el de Hoogovens (Holanda) y el de Thyssen (Alemania), que emplean esta tecnología (Tabla IV).

En el HA-6 de Hoogovens, con un sistema de distribución de carga Paul Wurth por canaleta móvil, al operar con consumo de coque de 270-290 $\mathrm{kg} / \mathrm{ta}$, que incluye $35-40 \mathrm{~kg} / \mathrm{ta}$ de menudos de coque, la proporción volumétrica de éste en el centro es del $90-100 \%$, y en la periferia del $25-40 \%$ (11). Hacia el centro se ubica cerca del $35 \%$ de la cantidad total de coque, en la zona intermedia del 35 al $43 \%$, y en la pared del 22 al $30 \%$. La disminución del consumo de coque desde $350 \mathrm{~kg} / \mathrm{ta}$ hasta los niveles señalados ocasiona una notable disminución del grosor de las capas de coque (desde 480 hasta $340 \mathrm{~mm}$ ). Los menudos de coque se cargan mezclados con el mineral en la zona periférica, para evitar el descenso de la base de la zona cohesiva.

El HA-1 de Thyssen, con un sistema de distribución de carga similar al horno anterior, logra índices elevados de operación con la siguiente distribución de materiales: en la periferia se ubica aproximadamente el $80 \%$ de la carga mineral y el $20 \%$ de la carga de coque; en el centro el $10 \%$ de la carga y $90 \%$ del coque. Esto corresponde a una carga de $100 \mathrm{~kg}$ de coque por tonelada de mineral en la 
periferia, y de aproximadamente $2.000 \mathrm{~kg}$ de coque por tonelada de mineral en el centro (12).

\subsection{Inyección de combustible en el crisol}

El volumen de combustible convencional que se consumía en el mundo a finales de los años 80 era del orden de $9 \times 10^{9}$ t. Se estima que para el año 2000 esta cifra se incrementará hasta $25 \times 10^{9}$ t. Más del $90 \%$ de las reservas energéticas existentes tienen su origen en fuentes minerales. A nivel mundial, cerca del $95 \%$ de la energía se obtiene a partir del uso de combustibles de origen orgánico y mineral. A finales de este siglo, se prevé que el combustible de origen orgánico quedará como la principal y determinante fuente de energía.

El costo elevado de la construcción y reconstrucción de las baterías de coquización, el incremento de los gastos de protección del medio ambiente durante la fabricación del coque, así como la paulatina reducción de los recursos de carbones coquizables, llevan a la necesidad de reducir el consumo de coque en el horno alto mediante su sustitución por combustibles auxiliares. Por otra parte, una característica común de la industria del coque y sus subproductos es el envejecimiento de sus instalaciones. Actualmente, la edad media de los hornos de coquería es de 18-23 años (13).

Con tal "escenario del coque", la idea de inyectar por toberas distintos combustibles auxiliares es un tema de actualidad entre los siderúrgicos. En calidad de combustible complementario, introducido en el chorro de viento, se utiliza gas natural y de batería, fuel oil, a veces alquitrán, y carbón (pulverizado o granulado). En la figura 3 se muestra un dispositivo para la inyección de combustibles auxiliares por toberas. Los caudales medios de inyección de estos combustibles, por países, se incluyen en la tabla III.

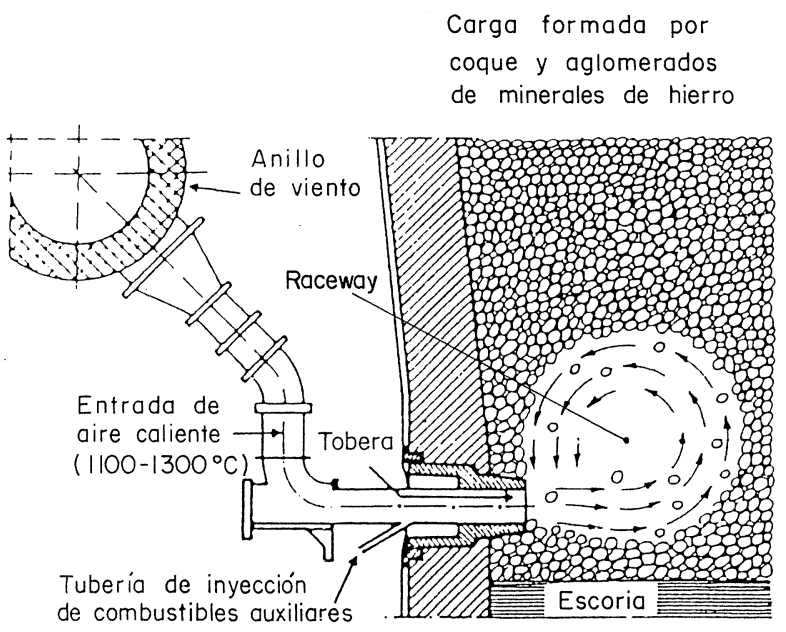

FIG. 3.-Zona de toberas del horno alto.

FIG. 3.-Blast furnace tuyeres zone.

\subsubsection{Combustibles gaseoso y líquido}

La inyección de gas natural es importante en los países con reservas de este combustible (EE.UU. y la antigua URSS), sin embargo, en los países de la UE y en el Japón este combustible no tiene aplicación en el horno alto, principalmente por la ausencia de grandes reservas.

En la industria siderúrgica la mayor demanda de fuel oil y de otros derivados del petróleo se observa en la República Federal Alemana, Japón y Francia a comienzos de los años 70, aunque en menor escala también se aplicó la inyección de combustible líquido en hornos de EE.UU, Canadá, Reino Unido, España, etc. Sin embargo, entre 1973-1975 la crisis energética mundial provocó un aumento de los precios del petróleo de 3-4 veces y, hacia 1982, de 1518 veces con respecto al precio previo a la crisis. Como resultado, al final de los años 70 e inicio de los 80 la utilización de fuel en el horno comienza a reducirse drásticamente. En la actualidad, en la UE sólo Luxemburgo, Austria, Finlandia y Alemania mantienen un determinado nivel de inyección, aunque se prevé que tienda a disminuir.

Debido a que se mantiene el crecimiento de los precios del gas y petróleo, en los últimos años tiene mayor difusión y desarrollo la inyección de carbón.

\subsubsection{Combustible sólido}

A nivel mundial las reservas de combustible sólido constituyen más del $80 \%$ de las reservas exploradas de combustible. Sin embargo, a pesar de estas grandes reservas los recursos en carbones coquizables son muy limitados. Así, por ejemplo, en uno de los mayores yacimientos de carbón (la cuenca hullera del Donbass, en Ucrania), sólo el $15,7 \%$ es coquizable. La escasez de carbones coquizables ocasiona una disminución de su proporción en la carga de las baterías de coque, por debajo de los límites permisibles, lo que a su vez reduce drásticamente su resistencia mecánica, al mismo tiempo que aumenta su precio.

Anteriormente, las desventajas del carbón no coquizable como combustible se debían a un coste relativamente alto de extracción (en comparación con los combustibles gaseoso y líquido), al contenido de azufre, presencia de ceniza y a la dificultad en el transporte neumático (14).

En la actualidad, la competitividad del carbón ha aumentado notablemente, lo que se explica principalmente por su menor coste en comparación con el petróleo y el gas natural. Según el Departamento de Energía de EE.UU. (15), para el período 1990-2005 se producirá el siguiente crecimiento de los precios de los combustibles usualmente empleados en la industria siderúrgica, en dólares EE.UU./Mcal: fuel 
oil desde 13 hasta 26,6; gas natural desde 14,5 hasta 29,0 y carbón no coquizable desde 7,5 hasta 9,7.

Por otra parte, las reservas de carbón mineral sobrepasan considerablemente las de otros combustibles orgánicos. A finales de 1990 las reservas de carbones no coquizables en el mundo eran del orden de $1.079 \times 10^{9} \mathrm{t}$, las de petróleo crudo de $136,5 \times 10^{9} \mathrm{t}$ y las de gas natural de $119,4 \times 10^{9} \mathrm{~m}^{3}$ (16). Para los niveles actuales de extracción, estas reservas pueden satisfacer la demanda de la industria siderúrgica durante 450 años en el caso de los carbones, de 35 a 45 años en el caso del petróleo y de 50 a 57 para el gas natural.

La inyección de carbón en Europa Occidental y en Japón comenzó en 1981. En 1983, en estos países operaban 5 hornos con esta tecnología, y en 1985 ya eran 10. La tendencia de aplicación de esta tecnología en la UE se muestra en la figura 4.

Se debe destacar que en el último decenio disminuyó notablemente el número de hornos en explotación, sin embargo, aunque la cantidad de hornos que se han equipado con sistemas de preparación e inyección de carbón se ha incrementado en sólo 8 unidades, se observa un considerable incremento de la proporción de hornos equipados con ICP (desde un $29 \%$ en 1987 hasta el $50 \%$ en 1995).

Los niveles máximos de inyección no se han establecido aún, aunque existe un aumento progresivo de la tasa de inyección. Por ejemplo, al estudiar la experiencia de los años 1960-1970, en el horno alto Amanda, de AK Steel (EE.UU.), una de las pioneras en esta tecnología, el caudal de carbón inyectado era de $58,5 \mathrm{~kg} / \mathrm{ta}(12,5 \%$ del combustible total). A inicios de los 80, la ICP era de $112 \mathrm{~kg} / \mathrm{ta}$ ( $25 \%$ del combustible total) y a comienzos de los 90 se inyectaban $157 \mathrm{~kg} / \mathrm{ta}$ para una sustitución del $35 \%$.

El rápido aumento de la cantidad de $\mathrm{CP}$ inyectado debido a las mejoras en la operación del horno,

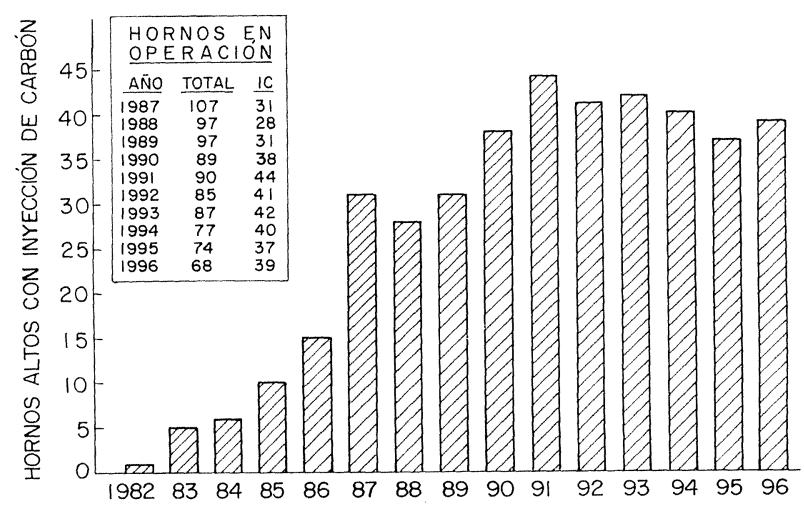

Fig. 4.- Hornos altos con inyección de carbón en la UE.

FIG. 4.- Blast furnaces with coal injection in the EU. es una característica de los hornos altos de Ilva, en Taranto (Italia), donde se ha asimilado esta tecnología en unos pocos años, según se puede apreciar en la tabla VIII.

En las tablas IV, VIII y IX se incluyen datos de operación de los hornos altos de la UE que utilizan inyección de carbón durante 1995.

Se puede ilustrar la efectividad de la inyección de caudales elevados de CP con el HA-6 de Hoogovens, en IJmuiden (Holanda), que durante 5 y 2 meses operó con caudales de 190 y $210 \mathrm{kgCP} / \mathrm{ta}$, respectivamente (11). En la tabla $\mathrm{X}$ se incluyen algunos índices de su operación en el período señalado. Durante toda la etapa experimental, el horno trabajó con estabilidad, el consumo de coque disminuyó linealmente al aumentar el caudal de CP, y el coeficiente de sustitución se mantuvo igual a 1,08 $\mathrm{kg}$ coque/kg carbón inyectado.

En la actualidad, se puede considerar como una práctica usual operar el horno con un consumo de coque de $320 \mathrm{~kg} / \mathrm{ta}$ mediante una tasa de ICP de $180 \mathrm{~kg} / \mathrm{ta}$ (17). En las fábricas de Dunkerque (Francia), IJmuiden (Holanda) y Schwelgern (Alemania) se ejecutan programas para incrementar la inyección de carbón hasta $250 \mathrm{~kg} / \mathrm{ta}$. Los mayores caudales de ICP logrados en Europa son:

Hoogovens HA-6 ... $212 \mathrm{~kg} / \mathrm{ta}$ nov. - dic. de 1992 Hoogovens HA-7 ... $210 \mathrm{~kg} / \mathrm{ta}$ ene. - mar. de 1991 Scunthorpe HA-

Queen Anne ...... 210 kg/ta sep. 1991 - feb. 1992

Scunthorpe HA-

Queen Anne ...... $210 \mathrm{~kg} / \mathrm{ta}$ ago. - nov. de 1993 Thyssen HA-1 ....... $200 \mathrm{~kg} / \mathrm{ta}$ oct. - nov. de 1992

Sollac HA-4 .......... 194 kg/ta may. - jun. de 1992

En diciembre de 1995, en España operaban 6 hornos altos con una producción de 5,5 × $10^{6}$ ta/año, utilizando fuel oil como combustible auxiliar. Dentro del programa de remodelación y modernización de la industria siderúrgica española, se prevé su sustitución por dos hornos altos de $2.348 \mathrm{~m}^{3}$ de volumen útil (cada uno), de $11,3 \mathrm{~m}$ de diámetro del crisol equipados con 29 toberas. Estos hornos operarán con $1.200{ }^{\circ} \mathrm{C}$ de temperatura del viento (enriquecido con un $2 \% \mathrm{O}_{2}$ ). La carga estará compuesta por $40 \%$ pelets y $60 \%$ sínter. Ambos hornos operarán con ICP en caudal de 175-190 $\mathrm{kg} / \mathrm{ta}$, lo que permitirá reducir el consumo de coque hasta 310-340 kg/ta; el sistema de inyección de carbón está constituido por dos instalaciones de 43,2 $\mathrm{t} / \mathrm{h}$. El primero de estos hornos fue puesto en marcha el 2 de octubre de 1996. A continuación se inicia la construcción del segundo horno, cuya puesta en marcha se prevé para diciembre de 1997. La capacidad de producción total de estos hornos será de $3,8 \times 10^{6}$ t/año y la inversión se ejecuta con un costo de 47.200 millones de pesetas. 
TABLA VIII.- Hornos altos en funcionamiento en Taranto (Italia)

TABLE VIII.- Operating blast furnaces in Taranto (Italy)

\begin{tabular}{|l|c|cccc|c|}
\hline \multirow{2}{*}{ Características } & \multirow{2}{*}{ Año } & \multicolumn{5}{|c|}{ Horno alto } \\
\cline { 3 - 7 } & & Núm. 1 & Núm. 2 & Núm. 4 & Núm. 5 & Media \\
\hline Diám. de crisol, m & & 10,6 & 10,3 & 10,6 & 14,0 & 11,4 \\
Volumen útil, m & & 2.117 & 1.971 & 2.032 & 3.650 & 2.443 \\
Núm. de toberas & & 27 & 25 & 27 & 36 & 29 \\
Inyección de carbón & 1991 & 0 & 56 & 28 & 6 & 22,5 \\
pulverizado, kg/ta & 1992 & 117 & 148 & 139 & 103 & 127 \\
& 1993 & 116 & 174 & 164 & 116 & 143 \\
& 1994 & 153 & 187 & 175 & 129 & 161 \\
& 1995 & 159 & 170 & 150 & 134 & 153 \\
Consumo de coque, & 1991 & 420 & 439 & 412 & 420 & 423 \\
$\mathrm{~kg} / \mathrm{ta}$ & 1992 & 353 & 337 & 332 & 361 & 346 \\
& 1993 & 366 & 332 & 320 & 354 & 340 \\
& 1994 & 334 & 309 & 324 & 357 & 331 \\
& 1995 & 335 & 332 & 346 & 350 & 341 \\
Productividad, & 1991 & 2,40 & 2,32 & 2,50 & 2,48 & 2,43 \\
t/m ${ }^{3} \cdot 24 \mathrm{~h}$ & 1992 & 2,50 & 2,69 & 2,66 & 2,44 & 2,57 \\
& 1993 & 2,48 & 2,82 & 2,69 & 2,45 & 2,61 \\
& 1994 & 2,21 & 2,84 & 2,67 & 2,41 & 2,53 \\
& 1995 & 2,53 & 2,70 & 2,61 & 2,57 & 2,60 \\
\hline
\end{tabular}

TABLA IX.- Parámetros de operación de los hornos altos con inyección de carbón en la UE en 1995 TABLE IX.- Parameters operation of blast furnaces with coal injection in European Community in 1995

\begin{tabular}{|l|c|c|c|c|c|}
\hline \multirow{2}{*}{ Países } & \multicolumn{2}{|c|}{ Hornos en operación } & \multicolumn{3}{c|}{ Parámetros analizados } \\
\cline { 2 - 6 } & Total & $\begin{array}{c}\text { Con inyección } \\
\text { de carbón }\end{array}$ & $\begin{array}{c}\text { Carbón inyecta- } \\
\text { do, } \mathrm{kg} / \mathrm{ta}\end{array}$ & $\begin{array}{c}\text { Consumo de } \\
\text { coque, } \mathrm{kg} / \mathrm{ta}\end{array}$ & $\begin{array}{c}\text { Productividad, } \\
\mathrm{t} / \mathrm{m}^{3} \cdot 24 \mathrm{~h}\end{array}$ \\
\hline Alemania & 20 & 9 & 115 & 369 & 2,24 \\
Bélgica & 8 & 7 & 125 & 384 & 2,26 \\
Francia & 11 & 8 & 121 & 357 & 2,15 \\
Holanda & 2 & 2 & 144 & 355 & 2,67 \\
Italia & 5 & 4 & 153 & 341 & 2,60 \\
Reino Unido & 8 & 3 & 143 & 354 & 2,44 \\
Suecia & 4 & 4 & 82 & 381 & 2,57 \\
Total & 58 & 37 & 122,6 & 365,5 & 2,34 \\
Media UE & & & & & \\
\hline
\end{tabular}

Las condiciones decisivas para operar con éxito el horno con caudales elevados de ICP son: emplear materias primas férreas y coque de alta calidad, aplicar nuevos métodos de carga y distribución de los materiales para mantener condiciones satisfactorias gasodinámicas, preparación del carbón y utilización de medidas especiales para intensificar su combustión. Algunos de estos aspectos han sido tratados en ( 7,8 y 18$)$.

Actualmente, se ejecutan proyectos de forma conjunta por instituciones científicas y fábricas de la UE que están encaminados a un mayor desarrollo de esta tecnología, al incremento del caudal de inyección de carbón y a la disminución del 
TaBla X.- Resultados de ensayos industriales en el HA-6 de Hoogovens, en IJmuiden

TABLE X.- Industrial test results in the BF-6 Hoogovens, in IJmuiden

\begin{tabular}{|l|r|r|r|}
\hline \multirow{2}{*}{ Parámetros } & \multicolumn{3}{|c|}{ Períodos durante 1992} \\
\cline { 2 - 4 } & Jun.-Oct. & \multicolumn{1}{|c|}{ Nov. } & \multicolumn{1}{c|}{ Dic. } \\
\hline Productividad: & & & \\
- t/día & 6.055 & 6.125 & 5.850 \\
- $\mathrm{t} / \mathrm{m}^{3} \cdot 24 \mathrm{~h}$ & 2,60 & 2,63 & 2,52 \\
Consumo de combustible, kg/ta: & & & \\
- coque & 253 & 240 & 236 \\
- menudos de coque & 37 & 36 & 36 \\
- carbón pulverizado & 190 & 205 & 212 \\
Total & $\mathbf{4 8 0}$ & $\mathbf{4 8 1}$ & $\mathbf{4 8 4}$ \\
Viento: & & & \\
- caudal, $\mathrm{m}^{3} /$ min & 3.200 & 3.755 & 3.492 \\
- contenido $\mathrm{O}_{2}, \%$ & 24,8 & 25,7 & 26,6 \\
Contenido en el arrabio, \%: & & & \\
- silicio & 0,38 & 0,38 & 0,36 \\
- desviación Sí & 0,10 & 0,10 & 0,08 \\
Temperatura del arrabio, ${ }^{\circ} \mathrm{C}$ & 1.501 & 1.492 & 1.495 \\
Volumen de escoria, $\mathrm{kg} / \mathrm{ta}$ & 236 & 234 & 238 \\
\hline
\end{tabular}

consumo de coque hasta $250 \mathrm{~kg} / \mathrm{ta}$ o por debajo de esta cantidad (19 y 20).

\subsection{Régimen de escoria y calidad del arrabio}

Es conocido que la calidad del arrabio se determina por la cantidad y características de la escoria. El volumen de escoria producida en los hornos de la UE es de 223-303 kg/ta. En Suecia es de sólo $153 \mathrm{~kg} / \mathrm{ta}$ y en Luxemburgo $509 \mathrm{~kg} / \mathrm{ta}$ (Tabla III). El índice de basicidad de la escoria, expresado por la relación $\mathrm{CaO} / \mathrm{SiO}_{2}$, se encuentra en el rango de 1,05-1,20.

Los hornos altos de Suecia tienen un interés especial desde el punto de vista del régimen de escoria. Por ejemplo, el HA-2 de SSAB, en Luleâ, opera con $100 \%$ de pelets LKAB con adición de $30-35 \mathrm{~kg}$ de caliza y $50 \mathrm{~kg}$ de escoria de convertidor por tonelada de arrabio, con un volumen de escoria de $145 \mathrm{~kg} / \mathrm{ta}$ (21). El índice de basicidad de la escoria es 0,95 , el coeficiente de distribución del azufre $L_{\mathrm{s}}$ se encuentra entre 30 y 33 , el contenido de $\mathrm{MgO}$ es $18 \%$ y el de $\mathrm{Al}_{2} \mathrm{O}_{3}$ se situa entre el 11 y $12 \%$. El horno tiene una productividad elevada $\left(3,05 \mathrm{t} / \mathrm{m}^{3} \cdot 24 \mathrm{~h}\right)$, funde diariamente entre $3.600 \mathrm{y}$ $3.700 \mathrm{t}$ arrabio, con una composición, en tanto por ciento de: 4,63 C; 0,43 Si; 0,39 Mn; 0,03 P; 0,052 S y $0,11 \mathrm{Ti}$, a una temperatura de $1.472{ }^{\circ} \mathrm{C}$.

En los últimos tiempos, en este horno se ensayan nuevos pelets $\operatorname{LKAB}\left(0,4 \% \mathrm{MgO}, 1,3 \% \mathrm{SiO}_{2}\right.$, $62,7 \% \mathrm{Fe}$ ), cuya proporción en la carga es del 50 $\%$. Posteriormente, se prevé pasar completamente a la utilización de este tipo de pelets, disminuyendo el volumen de escoria hasta $120 \mathrm{~kg} / \mathrm{ta}$.

El coeficiente de distribución del azufre entre la escoria y el arrabio $L_{\mathrm{S}}=(\mathrm{S}) /[\mathrm{S}]$ no es alto (20-45), lo que determina que el contenido de azufre en el arrabio experimente un aumento a pesar de que no es muy elevado su contenido en los materiales componentes de la carga. La fabricación de arrabio con $0,030-0,045 \% \mathrm{~S}$ es una práctica habitual. Todo el arrabio elaborado en Alemania, Finlandia, Suecia y Portugal contiene entre 0,05 y $0,07 \%$ S, y sólo algunos hornos en Bélgica, Italia, Reino Unido y España producen arrabio con bajo contenido de azufre $(0,018-0,025 \%)$. El arrabio con elevado contenido de azufre se somete a desulfuración fuera del horno alto.

El contenido medio de fósforo en el arrabio es del 0,06-0,12 \%; en Finlandia y Suecia del 0,030$0,037 \%$. La excepción es Luxemburgo donde se produce arrabio con elevado contenido de fósforo $(1,48 \%)$.

El contenido medio del silicio es de 0,3-0,8\% para una temperatura del arrabio de $1.470-1.500{ }^{\circ} \mathrm{C}$, aunque en Austria se produce arrabio con un contenido del $1,0 \%$ Si a $1.430-1.445{ }^{\circ} \mathrm{C}$ y en Pont-aMousson (Francia) con $2,23 \% \mathrm{Si}$ a $1.460{ }^{\circ} \mathrm{C}$.

$\mathrm{El}$ contenído de manganeso oscila entre un 0,25 y $0,62 \%$; solamente los hornos en Salzgitter y Bremen (Alemania) fabrican arrabio con bajo contenido de manganeso $(0,11-0,12 \%)$. El contenido de titanio generalmente es del 0,02-0,07 \%, aunque en Alemania, en los hornos 1 y 4 de Thyssen, en Schwelgern, así como en los hornos de EKO, en Eisenhüttenst, producen arrabio con 0,11\% Ti. Los mayores contenidos de titanio se obtienen en los hornos 2 y 4 de Dunkerque (Francia) con 0,45 y $0,28 \%$, respectivamente.

\section{PROCESOS SIDERÚRGICOS ALTERNATIVOS}

La producción mundial de metal primario en procesos distintos al del horno alto, en forma de hierro esponja, lupias y metal líquido, es de cerca de $30 \times 10^{6}$ t/año y está centralizada en Latinoamérica y en el Oriente Medio. De $23 \times 10^{6} \mathrm{t}$ de prerreducidos fabricados por reducción directa a nivel mundial en 1993, en Alemania se fabricaron 0,182 $\times 10^{6} \mathrm{t}$ y en Suecia $0,095 \times 10^{6} \mathrm{t}(2)$.

A pesar de que en los procesos de reducción directa del hierro en la UE se produce menos del $0,3 \%$ del arrabio elaborado en hornos altos (en el mundo esta proporción es del $4 \%$ ), se trabaja intensamente en la elaboración de nuevos métodos. Esto se explica, por una parte, por la complejidad para cumplir las medidas de protección del medio ambiente por las industrias del coque y sinterización y, por otra, por las ventajas que presenta la reducción directa para producción en pequeña escala y que son: más flexibilidad en los dispositivos y equipamiento en las coyunturas del mercado, y posibilidad de unión con otras 
nuevas tecnologías, en particular, con la elaboración de desbastes planos de poco grosor.

En este trabajo no se pretende realizar un análisis puntual de los procesos de reducción directa. A ello van dirigidos muchos trabajos publicados en revistas especializadas. En la figura 5 se muestra, solamente, la tendencia general de los procesos de obtención directa de metal primario. Prácticamente, todos los esquemas modernos se basan en la utilización de combustible sólido, principalmente carbones energéticos. Se diferencian, fundamentalmente, en las vías de utilización del gas regenerado. Son preponderantes los procesos con reformado de los gases de salida del baño de fusión o de recirculación del gas en el agregado de reducción. Se elaboran procedimientos sobre el empleo de energía eléctrica para la fusión completa de los materiales (22).

Una de las variantes pronosticadas para el futuro de la industria siderúrgica, a partir de la obtención de metal líquido, se muestra en la figura 6. Según este esquema, para la fabricación de metal primario se utiliza un convertidor de tipo ciclón CCF (cyclone converter furnace) sobre el que se está trabajando en la actualidad (23). El metal líquido se obtiene directamente a partir de finos de mineral de hierro y carbón granulado. El proceso CCF consta de dos etapas, que se producen en un reactor: la prerreducción y fusión completa del mineral en el ciclón y la reducción final en baño líquido. El metal líquido primario se transforma en acero mediante la com-

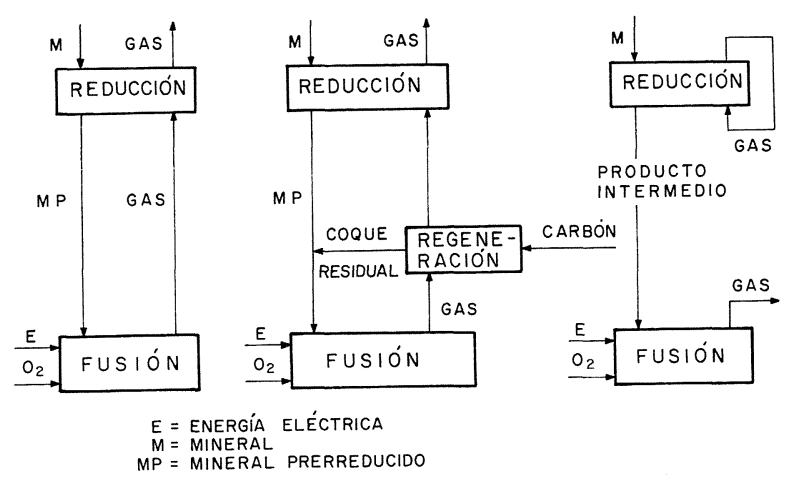

FIG. 5.- Diferentes procesos de obtención directa de metal primario.

\section{FIG. 5.- Different processes of direct obtaining of primary metal.}

bustión del carbono, silicio y la eliminación de las impurezas nocivas en reactores especiales.

El organismo alemán VDEh realiza un análisis comparativo entre los procesos de reducción directa y la producción en horno alto desde el punto de vista de utilización de la energía, de los índices económicos y de la interacción sobre el medio ambiente, llegando a la conclusión de que el horno alto mantiene su posición en las plantas siderúrgicas integradas.

El principal objetivo para aumentar la eficiencia del horno alto es la disminución del consumo de coque y la utilización de la energía de los gases de

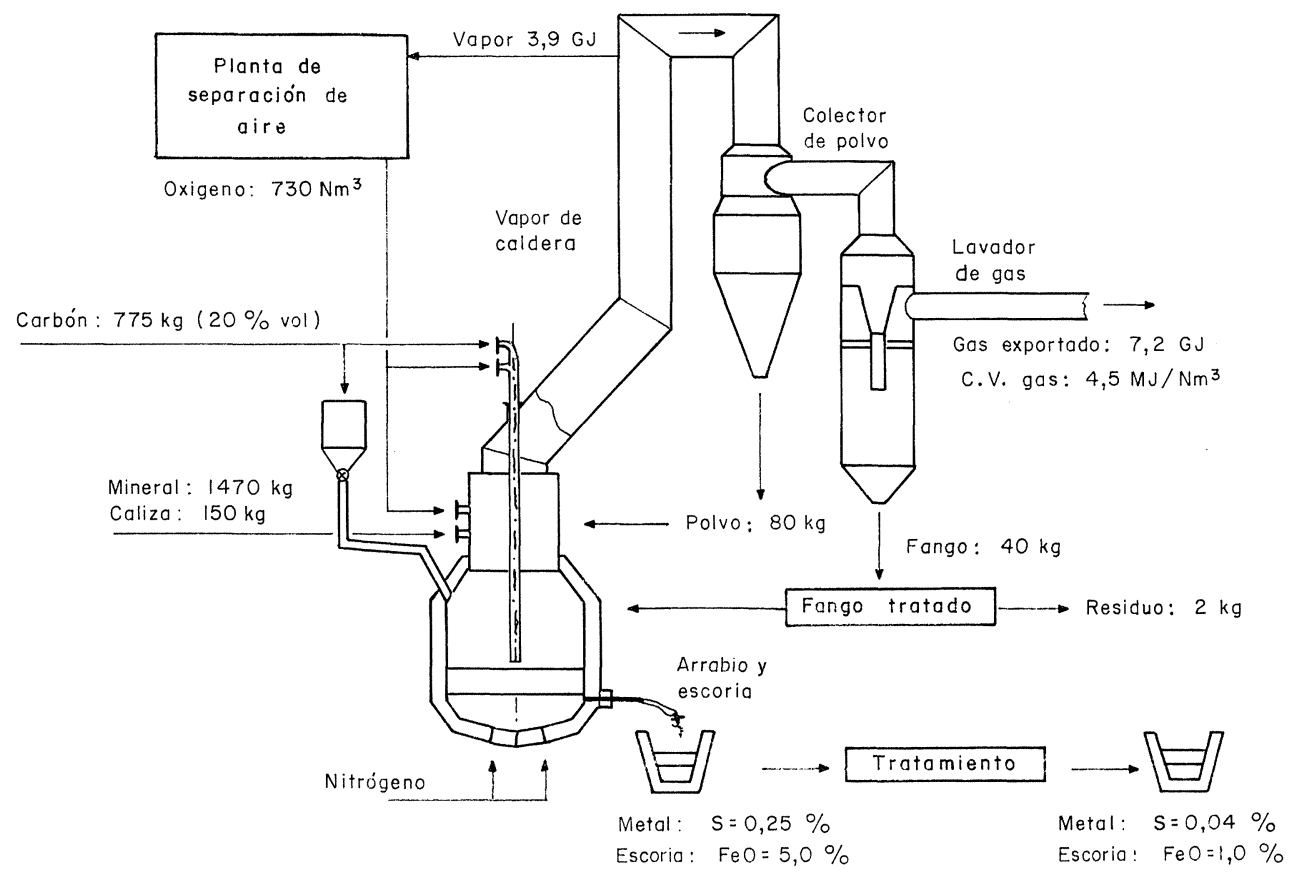

Fig. 6.- Esquema de flujo de una planta para fabricar $0,5 \times 10^{6}$ t/año de arrabio por el proceso CCF.

FIG. 6.-Flowsheet CCF ironmaking plant, $0.5 \times 10^{6} t / y$. 
salida (24). Será posible alcanzar este objetivo partiendo de la mejora en la preparación de la materia prima, la inyección de grandes cantidades de carbón y de materiales pulverulentos no combustibles y la obtención de gas del tragante reformado. La energía excedente del gas de horno será empleada en la obtención de vapor, oxígeno y energía eléctrica.

\section{CONCLUSIONES}

La fabricación de arrabio en la Unión Europea es del orden de $100 \times 10^{6}$ t/año, lo que constituye cerca del $20 \%$ de la producción mundial. El consumo de coque ha disminuido hasta 350-400 kg/ta, y algunos hornos durante varios meses han operado con consumos de coque inferiores a $300 \mathrm{~kg} / \mathrm{ta}$. La productividad de los hornos altos ha alcanzado 2,6$3,2 \mathrm{t} / \mathrm{m}^{3} \cdot 24 \mathrm{~h}$. Los elevados índices en el proceso del horno alto se han alcanzado mediante continuas mejoras de la calidad de la materia prima, aumento de la proporción de pelets en la carga, disminución del volumen de escoria hasta $220-300 \mathrm{~kg} / \mathrm{ta}$ (en algunos hornos hasta $150 \mathrm{~kg} / \mathrm{ta}$ ), inyección de carbón en caudales de 180-200 kg/ta, optimización de la distribución de los materiales y de la corriente gaseosa, y por la utilización de sistemas de control automático.

\section{REFERENCIAS}

(1) Steel Statistical Yearbook 1994. Committee on Statistics. Bruselas, 1995.

(2) World Steel in Figures. IISI, Association Internationale a but Scientifique. Bruselas. 1995 - 1997: 17.

(3) Minaev, A.A. y Konobalov, Yu.B. Metall Litio Ukraine, (11-12), 1995: 3-11.

(4) Babich, A.I., YaroshevskiI, S.L. y Tereshenko, V.P. Intensificación de la utilización de carbón pulverizado en el horno alto. Ed. Tejnika. Kiev, 1993: 191 (en ruso).

(5) Formoso, A., Garcia, L., Cores, A., Isidro, A., Babich, A. y YaroshevskiI, S. Rev. Minas, Oviedo, (13-14), 1996: $97-107$

(6) Operation data of sinter plants and blast furnace. European Blast Furnace Committee. Gijón (España), junio 1996.
(7) Babich, A., Yaroshevski, S., Garcia, L., Formoso, A., Cores, A., Isidro, A. y Ferreira, S. Rev. Metal. Madrid, 32 (2), 1996: 103-116.

(8) Babich, A., Yaroshevskit, S., Formoso, A., Isidro, A., Ferreira, S., Cores, A. y Garcia, L. ISIJ Int., 36 (10), 1996: 1.250-1.258.

(9) Steiler, J.M., Dufour, A. y Libralesso, J.M. Rev. Metall.-CIT, (10-11), 1995: 1.207-1.223.

(10) Uenaka, T., Miyatani, H., Hori, R., Norma, F., Shimizu, M., Kimura, Y. e InABa, S. Iron Steelmaker, 15 (11), 1988: 34-39.

(11) Koen, W., Toxopeus, H.L., Schoone, E.E., Vliet, C.v.d. y Berg, H.v.d. 53 rd Ironmaking Conf. Proc. Chicago, 1994: 429-436.

(12) Peters, K.H., Beppler, E. y Gerstenberg, B. Proc. 1st Int. Congress of Sci. and Tech. of Ironmaking. ISIJ. Sendai (Japón), 1994: 589-595.

(13) Nijhawan, B.R. Proc. 6th ILAFA-IVES Seminar Direct Reduction and Smelting. ILAFA. Ciudad Guyana (Venezuela), 1990: C1-C8.

(14) GASPER, D. Iron Steel Eng., 51 (7), 1974: 65-70.

(15) Mazar Marghall. R. Metall Bull. Monthly, (7), 1990: 67-68.

(16) JuKes, M.H. Met. Plant Tech., 16 (2), 1993: 53-60.

(17) Flierman, G.A., Langer, K., Leonard, D.C., LibraLesso, J.M., Massin, J.P. y Palomba, R. Proc. 3rd Intern. Ironmaking Congress. Gante (Bélgica), 1996: 64-71.

(18) BABICH, A.I., YAROSHEVSKII, S.L., KOCHURA, V.V. y TeresHENKO, V.P. Metall Litio Ukraine, (11-12), 1995: 12-18.

(19) Formoso, A., Isidro, A., Cores, A. y Ferreira, S. Further developments in blast furnace injection technology. ECSC Agreement No. 7210-AA/936. 1994.

(20) Formoso, A., Isidro, A., Cores, A. y Ferreira, S. Modelling of gas and char flows at high PCI through experimental and theoretical studies of the raceway and the dead man. ECSC Agreement No. 7210-AA/938. 1996.

(21) Hallin, M., Thulin, D. y TotTiE, M. 53 rd Ironmaking Conf. Proc. Chicago, 1994: 287-291.

(22) Lassat DE Pressigny, Y. Proc. ECSC Workshop Developments in Alternative Ironmaking Processes. Maizieresles-Metz, 1994: 63-64.

(23) Meijer, H.K.A., LaAr, J. Van, Knoop, W. Van Der, y Nederveen, R. van. Proc. 3rd Intern. Cokemaking Congress. Gante (Bélgica), 1996: 299-303.

(24) Gathergood, P.S. Proc. ECSC Workshop Developments in Alternative Ironmaking Processes. Maizieres-les-Metz, 1994: 75-80. 